\title{
Calibration-Free Volume Flow Measurement Principle Based on Thermal Time-of-Flight (TTOF) for Gases, Liquids and Mixtures
}

\author{
E. Engelien, O. Ecin, R. Viga, B. J. Hosticka and A. Grabmaier \\ University Duisburg-Essen, Institute of Electronic Components and Circuits, \\ Bismarckstraße 81, 47057 Duisburg \\ Eberhard.Engelien@uni-due.de, +49-203-379-2728
}

The use of mass and volume flow measurement techniques is essential in industries for process control purposes. Therefore, a novel flow measurement principle based on the Thermal Time-of-Flight (TToF) is applied with the methods of heat transfer including heat source and heat sinks. An appropriate flow meter is designed which can be deployed to any kind of fluid considering gases, liquids and mixtures. With this significant advantage the TToF flow meter is calibration-free.

The presented investigation includes a discontinuous heat source associated with a minimum of two thermal sensors arranged downstream. The heat source is used to generate a signal code [dT/dt] by injection of local thermal pulses into the flow line. The thermal sensors detect the time-dependent thermal gradients in the fluid at different positions. Herewith, the measurement technique aims at the determination of the flow velocity by ToF, with the particular advantage of applying to measurements of any fluids with unknown properties. The volume flow is determined by integrating the flow profile.

Keywords: Volume flow measurement, Thermal Time of Filght (TToF), signal processing, correlation technique

\section{Introduction}

The use of mass and volume flow measurement techniques is essential in industries for process control purposes, e. g. of gases and liquids. Conventional flow sensors are restricted to certain applications, all of which require different measurement principles. In recent years several different types of sensors were developed [2][12][17]. Depending on the specifications, for example metering precision, flow ratio, permitted decrease of pressure and also for the cost of production, different measurement principles are applied. The utilization of these measurement systems is mostly dependent on pressure, temperature, density, viscosity and homogeneity of the fluid. Hence, flow sensors must be calibrated and maintained for a certain application. At present, sensors cannot sufficiently measure fluids with unknown properties. This matter of fact necessitates new measurement techniques [17].

Thermal flow sensors are commonly used due to their low cost and simplicity. Their measurement method is currently based on detecting the heat displacement from a permanently heating element against the velocity of the passing fluid [3][6][12]. This kind of sensor is only applicable for fluids with known properties. Furthermore, ToF correlation flow sensors are already used for optic, acoustic and capacitive flow measurement techniques. However, these are restricted to the determination of multiphase flow with more than $10 \%$ available or injected signifiers in the flow. Problematical is that the measurement pattern formed by these signifiers constantly changes in between the flow sections [12].

Hence, the goal of this paper is to present a volume flow measurement technique applicable to any kind of fluid with unknown properties.

\section{Methods \& Materials}

In the following, the measurement technique for the detection of volume flow is presented as well as the associated experimental setup for the investigated media air, water vapour (air with $100 \%$ relative humidity) and water. Based on the measurement technique the theory of fluid mechanic and thermodynamics is discussed. The investigations are accompanied by numerical simulations.

\subsection{Measurement Theory}

The proposed measurement technique is based on the ToF of thermal signals [dT/dt] due to forced convection, predominating natural convection and conduction. TToF is based on the detection of single flow vectors within the flow profile. For this purpose a minimum of two thermal sinks (thermocouples) is required. Should temperature gradients as natural signals in the flow stream not be inherent in the medium, a signal generator in flow line (white dotted lines in Figure 1 and red dotted lines in Figure 2 and Figure 3 ) is to be applied.

In Figure 1 the propagation of a simulated thermal pulse in water at different stages is shown. The dotted lines describe the flow line in which the thermal signal is generated and measured. The TToF imaging sequence is a cutout of a pipe with a diameter of $\varnothing=1 "$. 


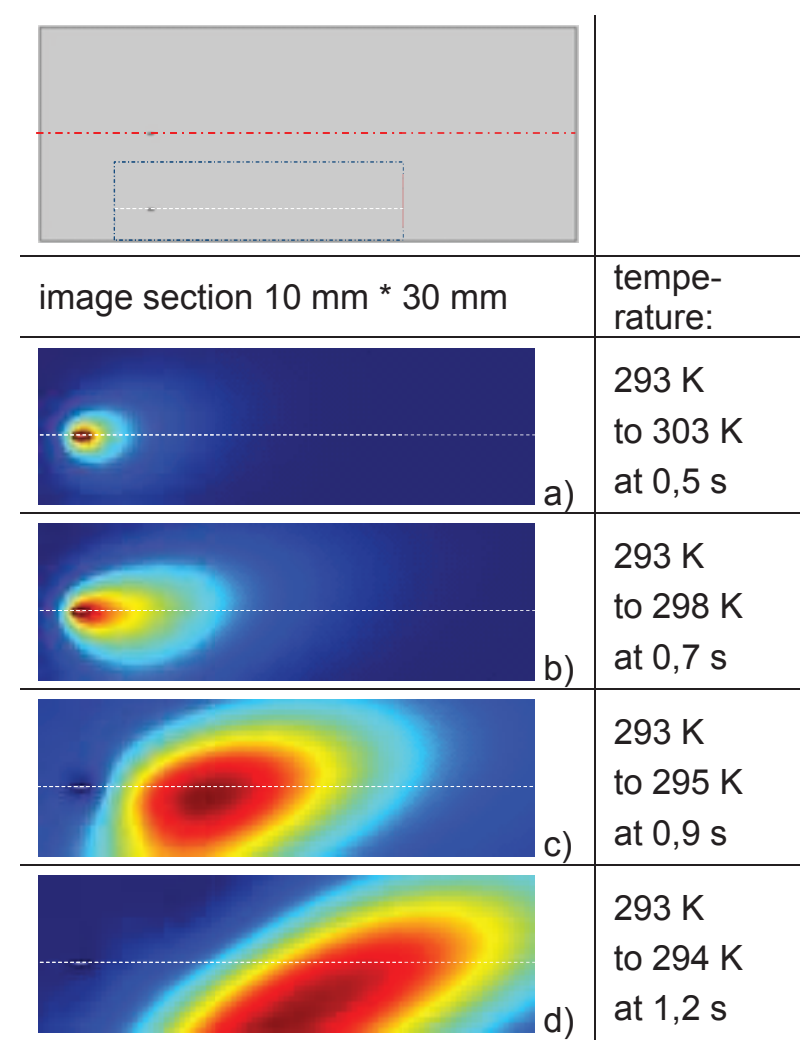

Figure 1: Temperature distribution for a heat pulse at the filament at the moment of formation and sequenced after every $0.2 \mathrm{~s}$ at a velocity of $v_{m}=0.05 \mathrm{~m} / \mathrm{s}$ [8].

This measurement technique can also be regarded as communication channel. In that case, the flowing medium in the pipe itself represents the transfer channel. The first signal sink detects the transmitted signal. The second sink, due to forced convection, detects the delayed, alleviated signal, which is transported by the flowing medium. This approach takes into account propagation of the thermal pulse due to conduction and natural convection [9].

\subsection{Experimental Setups}

The experimental setups were realised by a conveyer for the respective flowing medium, a reference flow sensor and the construction of specified sensors consisting of a filament to generate the necessary heat pulses and three thermocouples arranged downstream. Considering the pipe's diameter, the filament and the thermocouples are vertically plugged in the middle of the pipe.

The experimental setup is controlled by a CompactRio System (cRio-9024/9118) with LabView from National Instruments (NI). The voltage of the filament is controlled by the module 9263 and consists of sequent rectangular pulses with a period of $T=2 \mathrm{~ms}$ and a connect time $t=1 \mathrm{~ms}$. Thermal signals are detected by thermocouples at a frequency of $f_{D}=800 \mathrm{~Hz}$. Their output voltages are amplified by an operational amplifier AD595AQ from Analog Devices and detected by module cRio9234 (24 Bit of $\pm 5 \mathrm{~V}$ ) with a resolution of $\Delta T=0.0005 \mathrm{~K}$.

\subsubsection{Sensor Model for Gas and Water Vapour Flow Measurement}

The sensor model for gas and water vapour flow measurement is composed of a filament (Fi) and three thermocouples (TCs) (Figure 1). The generated thermal heat signal of $\Delta T=10 \mathrm{~K}$ is excited by 5 pulses, totally $50 \mathrm{mWs}$. The dimensions of the sensor model are a diameter of $\varnothing=40.0 \mathrm{~mm}$ of the pipe, $0.2^{*} 0.8 \mathrm{~mm}^{2}$ cross section for the filament and a diameter of $\varnothing=50 \mu \mathrm{m}$ for the type $\mathrm{K}$ thermocouples [11].

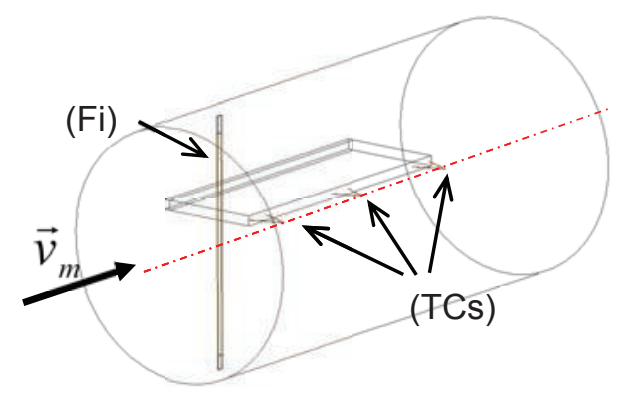

Figure 2: Sensor model with a filament $(\mathrm{Fi})$ and three thermocouples (TC).

The distance between the first $\mathrm{TC}_{1}$ to the second $\mathrm{TC}_{2}$ is $\Delta x_{12}=17.55 \mathrm{~mm}$, and from the first $\mathrm{TC}_{1}$ to the third $\mathrm{TC}_{3}$ is $\Delta x_{13}=35.5 \mathrm{~mm}$.

\subsubsection{Sensor Model for Water Flow Measurement}

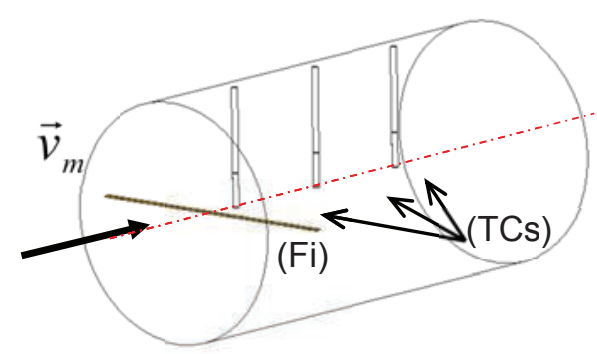

Figure 3: The construction of the water flow measurement consists of a filament and three isolated stick thermocouples.

The sensor model for water flow measurement consists of a pipe with a diameter of $\varnothing=1 "$, a filament with the dimension of $0.2^{*} 0.8 \mathrm{~mm}^{2}$ and three electrically isolated thermocouples type K (T.JC100-CAIN-IM025U Newport Omega). The generated thermal heat signal of $\Delta T=60 \mathrm{~K}$ is excited by 30 pulses, totally $300 \mathrm{mWs}$. The TCs have a diameter of $\varnothing=25 \mu \mathrm{m}$ and the encapsulation of $\varnothing=250 \mu \mathrm{m}$. The distance between the first $\mathrm{TC}_{1}$ 
to the second $\mathrm{TC}_{2}$ is $\Delta x_{12}=7.1 \mathrm{~mm}$, and from the first $\mathrm{TC}_{1}$ to the third $\mathrm{TC}_{3}$ is $\Delta x_{13}=21.2 \mathrm{~mm}$.

\subsection{Theory of Fluid Mechanics and Thermodynamics}

The investigations were conducted in the laminar range up to $R e \approx 2300$, described by equation 1. In a pipe through which a medium flows, a flow profile is created after sufficient inlet flow. For $R e<2300$ according to equation (2) a laminar flow profile is formed (Figure 4). For $R e>2300$ a turbulent flow profile will be more and more formed, in which the velocity remains equal along the diameter and only decreases to zero at the boundary conditions of the pipe wall [7]. This underlines the necessity to detect two flow velocity vectors to control the behaviour of the flow profile.

$$
R e=\frac{v \cdot d \cdot \rho}{\eta},
$$

Re being the Reynolds-Number, $v$ the velocity $\left[\mathrm{m} \cdot \mathrm{s}^{-1}\right], d$ the pipe diameter $[\mathrm{m}], \rho$ the density $\left[\mathrm{kg} \cdot \mathrm{m}^{-3}\right]$ and $\eta$ the dynamic viscosity [mPa.s].

$$
v(r)=v_{\max } \cdot\left(1-\left(\frac{r}{R}\right)^{2}\right),
$$

where the radius-dependent velocity is $v(r)$ $\left[\mathrm{m} \cdot \mathrm{s}^{-1}\right]$, the maximum velocity at $r=0 \mathrm{~mm}$ is $v_{\max }$, and the radius of the pipe is $R$ [m].

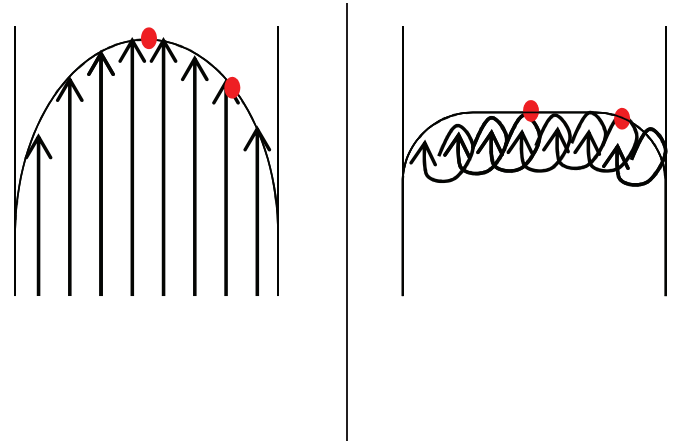

Figure 4: Profile of laminar and turbulent flow.

By introducing the filament and the thermocouples into the pipe, boundary layers $(\mathrm{BL})$ for flow and temperature (in case of the filament) are created (Figure 5 - equation (3)). The BLs are highly influenced already by small dimensions $(\mathrm{dx})$ of induced elements [16][18]. The effect does not significantly deepen with increasing size of the elements.

$$
\delta_{T}=\sqrt{\frac{x \cdot \lambda}{v_{m} \cdot \rho \cdot c_{p}}} \delta_{V}=\sqrt{\frac{x \cdot \eta}{v_{m} \cdot \rho}}
$$

where $\delta_{T}[\mathrm{~m}]$ and $\delta_{V}[\mathrm{~m}]$ is the boundary layer of temperature and flow with $x$ the width [m] of the injected element, $v_{m}$ the mean velocity $[\mathrm{m} / \mathrm{s}]$, $\lambda$ the specific thermal conductivity $\left[\mathrm{W} \cdot \mathrm{m}^{-1} \cdot \mathrm{K}^{-1}\right]$ and $c_{p}$ the specific thermal capacity $\left[\mathrm{J} \cdot \mathrm{kg}^{-1} \cdot \mathrm{K}^{-1}\right]$.

The elements are obstacles in the flow profile and lead to an interruption of the flow and in the turbulent section also to vortex. Nevertheless, the formation of the BLs of flow and temperature are also crucial properties for introducing heat pulses into the fluid - decreasing of the velocity by concurrently thermal coupling by conduction and natural convection. However, the velocity vector to be detected is demolished, for which applied $v=\mathrm{d} x / \mathrm{d} t=$ const. The change of the normalised velocity vector in flow line is shown in Figure 6 . Also demonstrated by Figure 6 is that in the described measurement model the detected value of the vellocity decreases to $65 \%$ of the initial value.

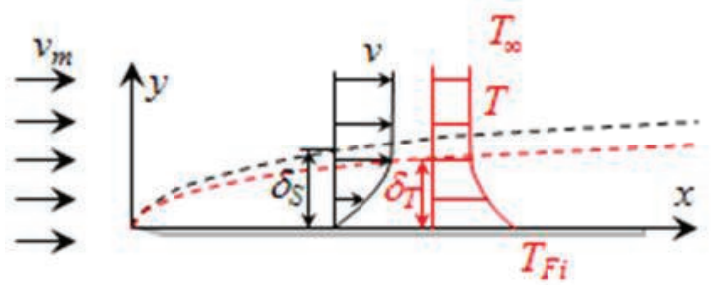

Figure 5: The boundary layer of temperature $\delta_{T}[\mathrm{~m}]$ and flow $\delta_{V}[\mathrm{~m}]$ due to the width of the filament or the length $/[\mathrm{m}]$ of the pipe.

The balance of $\Delta T$, the dimension intensity of the heat pulse, the velocity of the medium and the dimension of the fillament are described in equation (4) [18]. This equation determines the control loop for optimized heat pulses [14].

$$
\frac{\Delta Q}{\Delta t}=-\frac{\lambda \cdot A}{\Delta x} \cdot \Delta T,
$$

where $\Delta T$ is the temperature difference which drives a heat flow over a distance $\Delta x$ through an area $A$ in a time $\Delta t$.

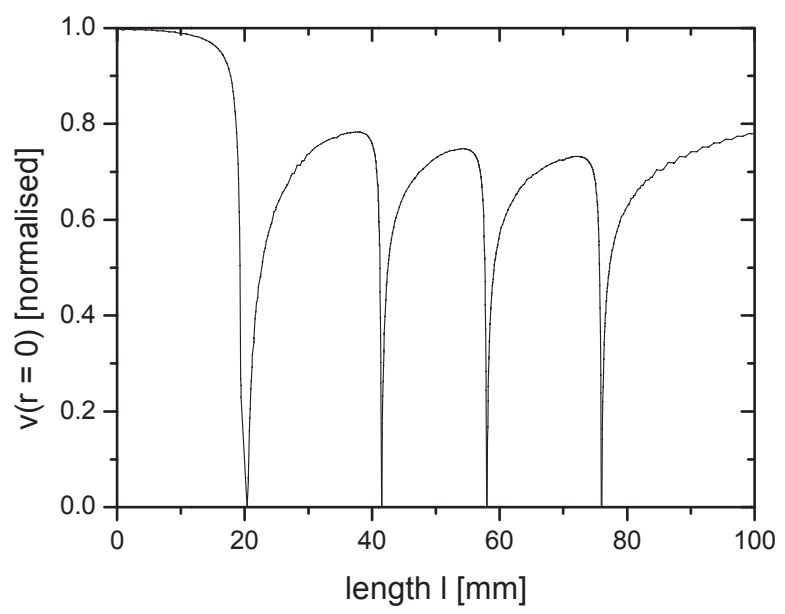

Figure 6: Normalised velocity vector value in flow line (red dotted lines in figure 2) [7]. 


\subsubsection{Natural \& Generated Signals}

For the applied signal processing (crosscorrelation) a time-dependent thermal signal $\mathrm{d} T / \mathrm{d} t$ is generally required. The signal communicated by the transmission channel needs to be mostly determined by forced convection. In terms of time-dependent propagation it needs to be differentiated from natural convection and conduction. For potential signal processing the signal has to exceed $\Delta T>0.01 \mathrm{~K}$ at the last downstream signal sink.

The generated heat signal could be e. g. sinus, rectangle, $\mathrm{PN}$-sequence or triangle. For the following investigation a sequence of rectangle pulses is applied.

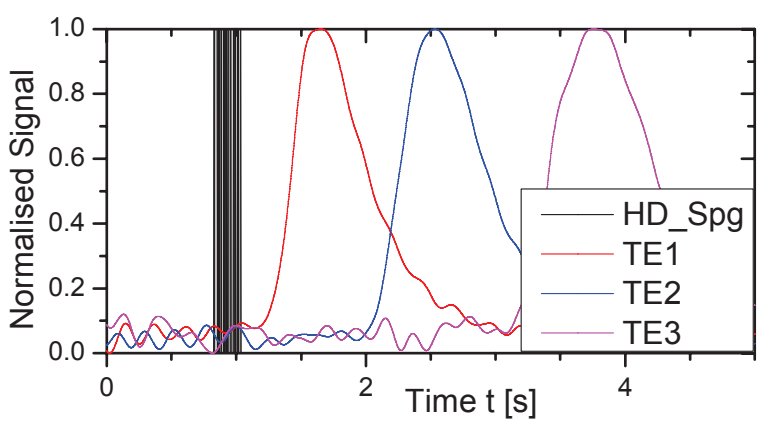

Figure 7: Normalised signal given to the filament and detected at the thermocouples for water $\left(v_{m}=0.017 \mathrm{~m} / \mathrm{s}\right)$.

\subsubsection{Signal Preprocessing}

Due to the construction of the thermocouples as closed loops, the detected thermal signals are influenced by diffusive and dispersive effects. These are on the one hand produced by thermal and electrical noise and on the other hand by electrical undesired signals. For this reason the direct time delay measurement does not constitute a suitable measurement signal analysis and processing method [19]. is:

The transfer function of the Chebyshev-filter

$$
G_{n}(\omega)=\frac{1}{\sqrt{1+\varepsilon^{2} T_{n}^{2}\left(\omega / \omega_{0}\right)}}
$$

$T_{n}$ being the Chebyshev-polynomial of the order $n$ with $n=3, \omega_{0}$ the cut-off frequency with $\omega_{0}=0.8 \mathrm{~Hz}$ and $\varepsilon$ the ripple factor $\varepsilon=10 \mathrm{~dB}$. The sampling factor is 80 .

The transfer function of the Elliptic / Cauerfilter is:

$$
G_{n}(\omega)=\frac{1}{\sqrt{1+\varepsilon^{2} R_{n}^{2}\left(\xi, \omega / \omega_{0}\right)}}
$$

$R_{n}$ being the Cauer-polynomial of the order $n$ with $n=3, \omega_{0}$ the cut-off frequency with $\omega_{0}=0.25 \mathrm{~Hz}, \varepsilon$ the ripple factor $\varepsilon=10 \mathrm{~dB}, \xi$ the selectivity factor $\xi=70 \mathrm{~dB}$. The sampling factor is 45 .

The induced thermal signals have a frequency $f_{i}<1 \mathrm{~Hz}$. For the signal filtering the Chebychev and the Cauer filter are therefore selected as they have an abrupt behaviour for specified cutoff-frequency [1]. The filters minimize the error between the idealized and the actual filter characteristic over the range of the filter, but with ripples in the passband.

For observing a time delay by signal processing the sensor signal needs signal conditioning. Therefore, the signal offset is corrected to null and normalised.

\subsubsection{Signal Processing}

For the calculation of the time delay of the thermal signal between the signal sinks (thermocouples) correlation techniques are used in the domain of signal processing.

Hence, the output signals of these thermocouples show change in amplitude and time behaviour. Since the output signals of the thermocouples are compared with regard to their time behaviour, the cross-correlation technique is applied [5]. Correlating the first output signal $x_{T 1}(t)$ with both, the second $x_{T 2}(t)$ and the third $x_{T 3}(t)$ signal, two cross-correlation functions $R x_{T 1} x_{T 2}(\tau)$ and $R x_{T 1} x_{T 3}(\tau)$ are obtained as a function of the time delay $\tau$ between these signals. The mathematical expression of the cross-correlation is given for two different signals $x(t)$ and $y(t)$ as:

$$
R_{x y}(\tau)=\frac{1}{T} \int_{0}^{T} x(t+\tau) \cdot y(t) d t
$$

where $T$ denotes the time length of the signal.

For discrete signals with the length of $N$ values the cross-correlation function yields [1]:

$$
R_{x y}(k)=\frac{1}{N} \sum_{n=0}^{N-1} x(n+k) \cdot y(n)
$$

where $k$ is the dependent sampling value [13].

Obtaining the cross-correlation coefficients of the thermocouples' output signals, the time delay between these signals is determined [15].

\section{Results \& Discussion}

The main target of the described investigations consists in the detection and characterisation of the propagation of heat pulses in the flow of air with $30 \%$ relative humidity, water vapour of $100 \%$ relative humidity and water at $20^{\circ} \mathrm{C}$ temperature. The measured heat pulses at the thermocouples had a temperature of $0.01 \mathrm{~K}<\Delta T<4 \mathrm{~K}$. Thereby the 
thermal and electric noise and the interfering signals were $\Delta T= \pm 0.2 \mathrm{~K}$.

In the investigation each velocity is measured seven times, values above $60 \%$ of the average velocity were neglected - mostly velocity values $>1 \mathrm{~m} / \mathrm{s}$. The measured range is for air flow $v_{\max }=0.01 \mathrm{~m} \cdot \mathrm{s}^{-1}-3.5 \mathrm{~m} \cdot \mathrm{s}^{-1}$ and for water $v_{\max }=0.006 \mathrm{~m} \cdot \mathrm{s}^{-1}-0.65 \mathrm{~m} \cdot \mathrm{s}^{-1}$. The calculated velocities (CV) are shown up to a $R e>2300$ (air flow $v_{\max }=1.9 \mathrm{~m} / \mathrm{s}$ and water flow $v_{\max }=0.18 \mathrm{~m} / \mathrm{s}$ ) where the flow profile is getting instable.

In the following, the results for the three tested media will be described. To demonstrate the results in Figure 8 - Figure 10 logarithmic scales are used. The results for air are shown in Figure 8. The velocities $v_{12}$ and $v_{13}$ show a linear behaviour to the referenced maximum velocity (RMV), whereas the velocity curve $v_{12}$ crosses the curve of $v_{13}$ at $v=0.11 \mathrm{~m} / \mathrm{s}$.

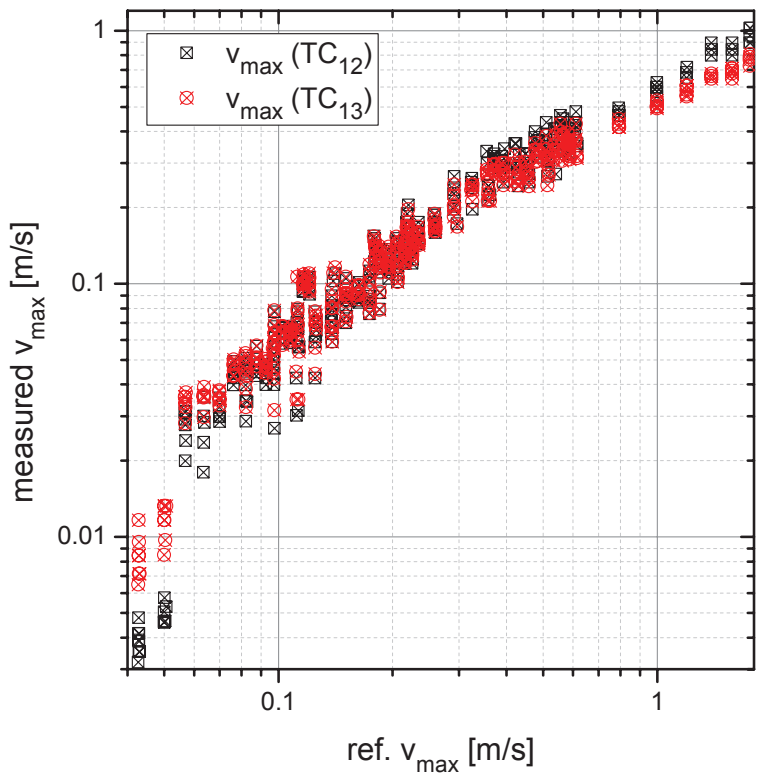

Figure 8: $\mathrm{CV}$ with correlation at a time delay of the signals $\mathrm{TC}_{1}-\mathrm{TC}_{2}$ (black/ $\square$ ) and $\mathrm{TC}_{1}-\mathrm{TC}_{3}$ $(\mathrm{red} / \Delta)$ in the middle of the pipe for air.

At velocities $0.09 \mathrm{~m} / \mathrm{s}, 0.12 \mathrm{~m} / \mathrm{s}$ and $0.7 \mathrm{~m} / \mathrm{s}$ a bend can be observed due to the fact that the conveyer was changed at these points. For RMV up to $1.8 \mathrm{~m} / \mathrm{s}$ the standard deviation is $<7 \%$. The standard deviation increases exponentially with increasing velocity values and for velocities of $3.5 \mathrm{~m} / \mathrm{s}$ it is $19 \%$. The measured values shown in Figure 8 deviate at first considerably from the expected values. At a RMV of $0.5 \mathrm{~m} / \mathrm{s}$ the values stabilise at $65 \%$ of the expected values. These results coincide with previous analysis on the basis of numerical simulations as shown in Figure 6.

In Figure 9 the results for water vapour are shown. A drift in smaller and higher RMV values can be observed due to the fact that the change of the conveyer has higher effect. This is combined to a $50 \%$ higher deviation from the averaged velocity compared to flowing air.

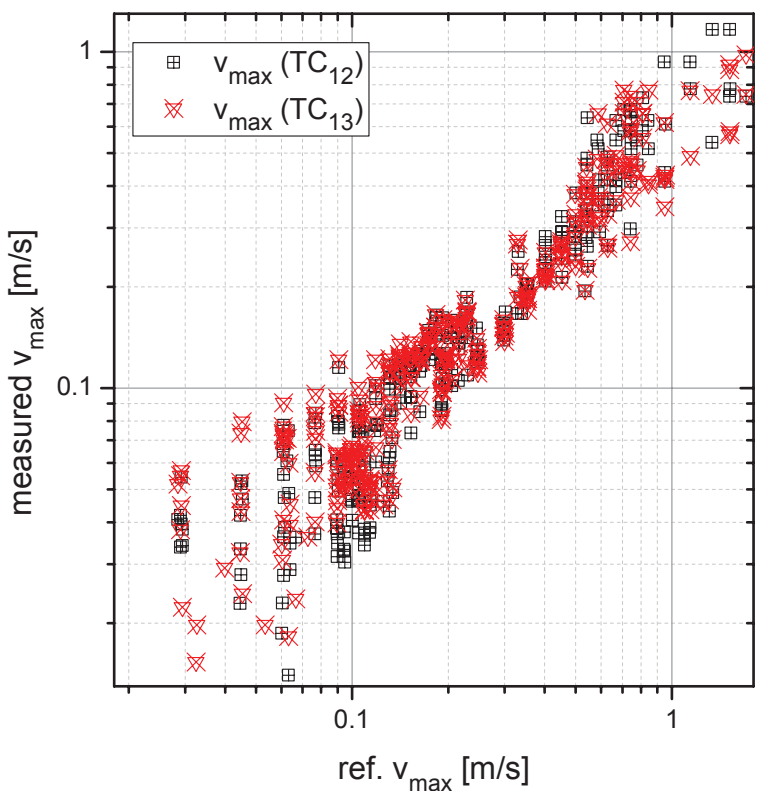

Figure 9: $\mathrm{CV}$ with correlation at a time delay of the signals $\mathrm{TC}_{1}-\mathrm{TC}_{2}$ (black/ $\square$ ) and $\mathrm{TC}_{1}-\mathrm{TC}_{3}$ $(\mathrm{red} / \Delta)$ in the middle of the pipe for water vapour.

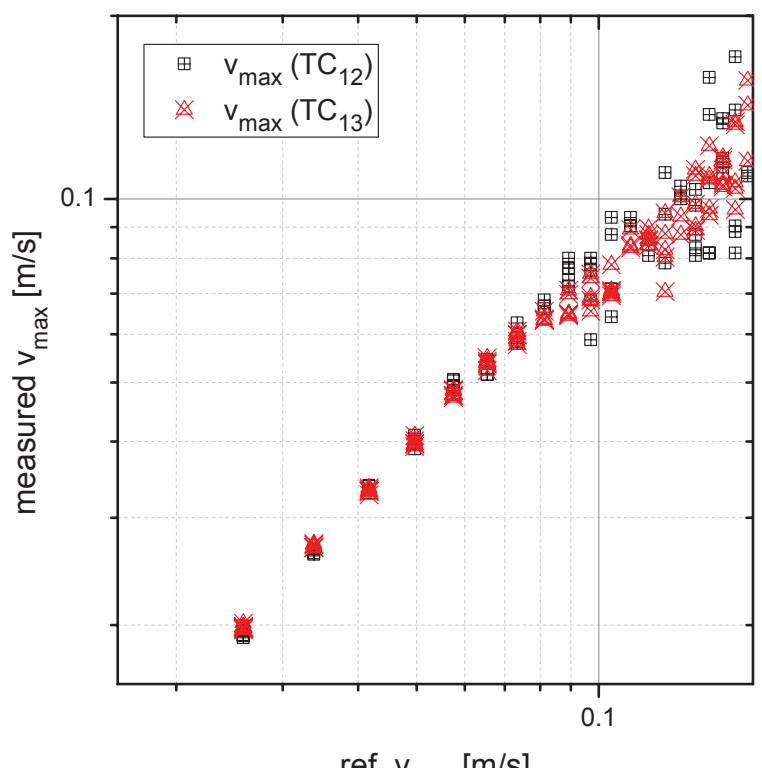

ref. $v_{\text {max }}[\mathrm{m} / \mathrm{s}]$

Figure 10: $\mathrm{CV}$ with correlation at a time delay of the signals $\mathrm{TC}_{1}-\mathrm{TC}_{2}$ (black/ $\square$ ) and $\mathrm{TC}_{1}-\mathrm{TC}_{3}$ $(\mathrm{red} / \Delta)$ in the middle of the pipe for water.

The results for water are shown in Figure 10. The CVs show a high linear behaviour to RMV. The standard deviation of CV in this case is up to RMV $v \approx 0.09 \mathrm{~m} / \mathrm{s}$ less than $1 \%$. For RMVs up to $0.12 \mathrm{~m} / \mathrm{s}$ the standard deviation for $\mathrm{CV}_{12}$ is $6 \%$ and for $\mathrm{CV}_{13}$ is $18 \%$. At a velocity of $0.65 \mathrm{~m} / \mathrm{s}$ the deviation is $25 \%$. The accuracy remains constant even if there are particle's in flowing media up to $1 \mathrm{~mm}$. 


\section{Conclusion}

In the present paper, a new technique for volume flow measurement was introduced. With the results outlined in 3 . it could be shown that the measurement technique is applied to air, water vapour and water and in particular it is expected to other fluids with unknown properties.

Especially for $R e<2200$ in the case of water the standard deviation is $<1 \%$ and for air $<7 \%$. This is partly due to the fact that for $R e>2000$ the signal disappears progressively in noise and interference signals and that signal pre-processing becomes more and more important. In this case mistakes in preprocessing, e.g. inappropriate choice and adjustment of the filter, lead to a drift of the signal centre, which causes up to $100 \%$ deviation of the calculated delay time.

Based on present investigations the measurement technique is object to the following limitations: one sensor distance measurement allows a velocity determination in a range of $1: 100$ and limited dissolution for velocities similar to the propagation of the natural convection $\left(v \approx 0.02 \mathrm{~m} \cdot \mathrm{s}^{-1}\right)$.

Future investigations will firstly lead to an enhanced sensor construction design to measure the designated velocity vector in the flow profile. Secondly, they will focus on preprocessing especially for $R e>2000$. Finally, further analysis of the thermal decay behaviour of the signals will be done via investigations on the time constant $\tau=c_{p} \cdot \lambda$ to draw conclusion from the flowing media.

\section{Acknowledgements}

This work is supported by the German research association (Deutsche Forschungsgemeinschaft, DFG) and is part of the sponsored project ANTEMES.

\section{Literature}

[1] Avirav, Y.; Guterman, H.; Ben-Yaakov, S.: "Implementation of digital signal processing techniques in the design of thermal pulse flowmeters", IEEE Transactions on Instrumentation and Measurement; Volume 39, Issue 5, pp. 761 - 766, 1990.

[2] Baker, R.C.: "Flow Measurement Handbook", Cambridge University Press, Cambridge, ISBN 521-48010-8, 2000.

[3] Benjamin, S.F.; Roberts, C.A.: "Measuring flow velocity at elevated temperature with a hot wire anemometer calibrated in cold flow". International journal of heat and mass tranfer, 45:703 706, 2005.
[4] Bernhard, F.: "Technische Temperaturmessung: Physikalische und messtechnische Grundlagen, Sensoren und Messverfahren, Messfehler und Kalibrierung": Springer-Verlag ISBN 3-540-62672-7, 2004.

[5] Borgioli, R. C.: "Fast Fourier Transform Correlation Versus Direct Discrete Time Correlation", Proceedings of the IEEE, Vol. 56, Issue 9, pp. 1602-1604, 1968.

[6] Cao, Y.; et all: "Flow Rate measurement System Using thermal Air Flow Sensor", $9^{\text {th }}$ ICEMI, IEEE 987-1-4244-4864-8, 2009.

[7] Durst, F.: "Grundlagen der Strömungsmechanik", Springer-Verlag, ISBN 3-540-313230,2006

[8] Ecin, O.; Engelien, E.; et all: "Modelling Thermal Time-of-Flight Sensor for Flow Velocity Measurement", COMSOL Conference 2009, Milan.

[9] Ecin, O.; Engelien, E.; et all: "Thermal signal behaviour for air flow measurements as fundamentals to Time-of-Flight", ESTC $20103^{\text {rd }}$, 13-16 Sept., ESTC.2010.5642983, Berlin.

[10] Engelien, E.; Ecin, O.; et all.: "Evaluation on Thermocouples for the Thermal Time-of-Flight Measurement", $54^{\text {th }}$ IWK, paper-id 87, pp. 1-5, IImenau, 2009.

[11] Engelien, E.; Ecin, O.; et all: "Sensor Modelling for Gas Flow Measurement using Thermal Time-of-Flight Method", 15th ITG/GMA-Fachtagung, Sensoren und Messsysteme 18.-19.05.2010, pp. 1-8, Nürnberg.

[12] Fiedler, O.: "Strömungs- und Durchflußmeßtechnik", R. Oldenbourg Verlag GmbH München, ISBN 3-486-22119-1, 1992.

[13] Frey, T.; Bossert, M.: "Signal- und Systemtheorie", Vieweg+Teubner Verlag, ISBN 978-3-8351-0249-1, 2008.

[14] Marek, R.; Nitsche, K.: „Praxis der Wärmeübertragung", Carl Hanser Verlag, ISBN 978-3-446-4099-6, 2007.

[15] Oliveira, A.; et all: "Compensation of the Fluid Temperature Variation in a Hot-Wire Anemometer", IEEE IMTC, Ottawa, May 19-21, pp. 1377-1380, 1997.

[16] Sigloch, H.: "Technische Fluidmechanik", Springer-Verlag Berlin Heidelberg, ISBN 978-3-540-44633-0, 2008.

[17] Spurk, J.H.; Aksel, N.: "Strömungslehre: Einführung in die Theorie der Strömungen", Springer-Verlag Berlin Heidelberg, ISBN 3-540-38439-1, 2007.

[18] van Leyen, D.: "Wärmeübertragung", Verlag Siemens AG Berlin, München, 1971.

[19] Xu, Y.; de Graaf, G.; Wolffenbuttel, R. F.: "DSP Cross-Correlator for Use in a Thermal Flow Meter", IEEE Instrumentation and Measurement Technology Conference, Vol. 1, pp. 519-522, 1996. 